\title{
Editorial
}

\section{Would Kraepelin reconsider the distinction between schizophrenia and bipolar disorder if he had access to recent molecular genetics evidence?}

Emil Kraepelin divided psychotic disorders into two distinct categories, dementia praecox and manicdepressive illness (1). This dichotomous classification has been maintained in the ICD and recently updated in DSM diagnostic systems, but there is clearly overlapping phenotypes between the diagnoses of schizophrenia and bipolar disorder as well as schizoaffective disorders (2). Further, several authors have argued for the continuum model, with prototypic bipolar disorder in one end and prototypic schizophrenia in the other (3). These models are all based on descriptive symptomatology and not on underlying mechanisms (4). Epidemiological studies have shown that schizophrenia and bipolar disorder run in families (5), and provided support for shared genetic risk factors. Twin studies have estimated heritability rates to be $\sim 80 \%$ for both disorders $(6,7)$. With the recent progress in technology in the genetics field, it has been proposed that molecular genetics may help inform the nosology of severe mental disorders.

The microarray genotyping technology has provided a boost to psychiatric genetics. This has enabled genotyping of unprecedented large samples $(6,8)$, and facilitated by the international Psychiatric Genomics Consortium (PGC; https://pgc.unc.edu/ index.php), a series of recent large genome-wide association studies (GWAS) has been conducted. These have identified several new common risk variants [single nucleotide polymorphisms (SNPs)] associated with psychotic disorders $(9,10)$. A recent PGC cross-disorder mega-analysis $(n=33332$ cases, $n=27888$ controls) investigating schizophrenia, bipolar disorder, major depressive disorder, autism spectrum disorders and attention-deficit hyperactivity disorder identified common risk variants (SNPs) for all five disorders (11). Among these disorders, the strongest overlap in genetic risk was observed between schizophrenia and bipolar disorder. In addition to common variants (SNPs), rare structural variants called copy number variants (CNVs) have been found associated with schizophrenia (12). Interestingly, CNVs seem not to be associated with bipolar disorder to the same degree (13). Thus, there is evidence for shared as well as disease-specific genetic risk for schizophrenia and bipolar disorder. This supports the hypothesis of partial genetic overlap between the two disorders, which was the rationale for the study by $\mathrm{Gu}$ et al. (14) In this issue of the journal. Gu et al. performed a meta-analysis of eight studies with schizophrenia $(n=2953$ cases, $n=3153$ controls) and six studies of bipolar disorder ( $n=923$ cases, $n=928$ controls), focusing on the $1354 \mathrm{C} / \mathrm{T}$ genetic polymorphism of 5-hydroxytryptamine receptor $2 \mathrm{~A}$. In their samples with both Caucasian and Asian ethnicity, they did not find any association with this gene marker for neither schizophrenia nor bipolar disorder. This negative finding is in line with the concept of overlapping genetic risk factors in these disorders.

However, the single SNP approach is not able to capture much of the heritability of these disorders. Recently, new evidence for a polygenic architecture of bipolar disorder and schizophrenia was presented. The first evidence for a polygenic overlap in psychotic disorders came from a combined GWAS (6), where the cumulative risk from the entire genome is extracted from one GWAS dataset to predict risk in an independent sample (6). As schizophrenia and bipolar disorder share common genetic risk, we have applied new statistical methods to leverage the pleiotropy between the two disorders to improve gene discovery, that is, use information on risk variants identified in one disorder to improve detection of risk variants in the other (15). Based on these Bayesian methods, it is also possible to estimate the 
total number of SNPs associated with these disorders. These analyses suggest that there are $\sim 12000$ SNPs associated with both schizophrenia and bipolar disorders. Further, these new statistical methods for analysing GWAS results of polygenic complex disorders can also be used to identify potential common biological pathways, as illustrated with schizophrenia and cardiovascular disease risk factors (16).

The advance in our understanding of the underlying genetic architecture of bipolar disorder and schizophrenia may have a large potential for generating new knowledge about the pathophysiology of these major neuropsychiatric disorders which may eventually also lead to better diagnostic systems as well as new drug development.

$$
\begin{array}{r}
\text { Ole A. Andreassen } \\
\text { NORMENT, K.G. Jebsen Centre for Psychosis } \\
\text { Research, Institute of Clinical Medicine, University } \\
\text { of Oslo and Division of Mental Health and } \\
\text { Addiction, Oslo University Hospital, Oslo, Norway } \\
\text { E-mail: o.a.andreassen@medisin.uio.no }
\end{array}
$$

\section{References}

1. BeER MD. Psychosis: a history of the concept. Compr Psychiatry 1996;37:273-291.

2. Simonsen C, Sundet K, Vaskinn A et al. Neurocognitive dysfunction in bipolar and schizophrenia spectrum disorders depends on history of psychosis rather than diagnostic group. Schizophr Bull 2011;37:73-83.

3. Craddock N, Owen MJ. The Kraepelinian dichotomy - going, going... but still not gone. Br J Psychiatry 2010;196:92-95.

4. KendLer KS. An historical framework for psychiatric nosology. Psychol Med 2009;39:1935-1941.

5. Lichtenstein P, Yip BH, Bjork $\mathrm{C}$ et al. Common genetic determinants of schizophrenia and bipolar disorder in
Swedish families: a population-based study. Lancet 2009; 373:234-239.

6. Purcell SM, Wray NR, Stone JL et al. Common polygenic variation contributes to risk of schizophrenia and bipolar disorder. Nature 2009;460:748-752.

7. Smoller JW, Finn CT. Family, twin, and adoption studies of bipolar disorder. Am J Med Genet C Semin Med Genet 2003;123C:48-58.

8. Stefansson H, Ophoff RA, Steinberg $\mathrm{S}$ et al. Common variants conferring risk of schizophrenia. Nature 2009; 460:744-747.

9. Ripke S, Sanders AR, Kendler KS et al. Genome-wide association study identifies five new schizophrenia loci. Nat Genet 2011;43:969-976.

10. SkLAR P, RipKe S, ScotT LJ et al. Large-scale genomewide association analysis of bipolar disorder identifies a new susceptibility locus near ODZ4. Nat Genet 2011;43: 977-983.

11. Psychiatric Genomics Consortium Cross Disorders Group. Identification of risk loci with shared effects on five major psychiatric disorders: a genome-wide analysis. Lancet 2013;381:1371-1379.

12. Stefansson H, Rujescu D, Cichon S et al. Large recurrent microdeletions associated with schizophrenia. Nature 2008;455:232-236.

13. Bergen SE, O'Dushlaine CT, Ripke S et al. Genome-wide association study in a Swedish population yields support for greater $\mathrm{CNV}$ and $\mathrm{MHC}$ involvement in schizophrenia compared with bipolar disorder. Mol Psychiatry 2012;17: 880-886.

14. Gu L, TAN J, Su L et al. Meta-analysis of the HTR $2 A-1354 \mathrm{C} / \mathrm{T}$ polymorphism in schizophrenia and bipolar disorder. Acta Neuropsych 2013;25:311-319.

15. Andreassen OA, ThOMPSON WK, Schork AJ et al. Improved detection of common variants associated with schizophrenia and bipolar disorder using pleiotropyinformed conditional false discovery rate. PLoS Genet 2013;9:e1003455.

16. Andreassen OA, Djurovic $S$, Thompson WK et al. Improved detection of common variants associated with schizophrenia by leveraging pleiotropy with cardiovascular-disease risk factors. Am J Hum Genet 2013;92:197-209. 